\title{
Can we turn to our next page in nuclear cardiology?
}

\author{
Leslee J. Shaw, PhD, ${ }^{a}$ and Ami E. Iskandrian, $M D^{b}$ \\ a Emory University, Atlanta, Georgia \\ b Cardiology Department, UAB, Birmingham, Alabama
}

Received Mar 25, 2017; accepted Mar 27, 2017

doi:10.1007/s12350-017-0877-0

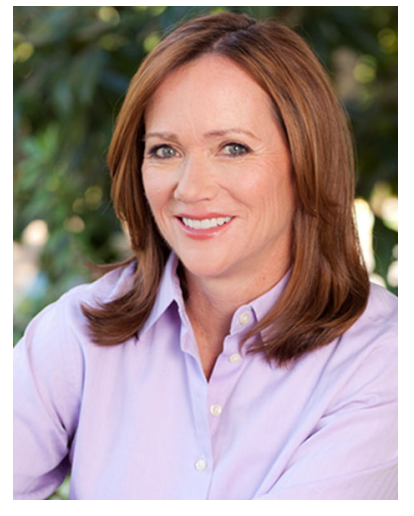

Leslee J. Shaw, Senior

Associate Editor,

Journal of Nuclear

Cardiology.

Nuclear cardiology has had an illustrious history with substantial success in developing high-quality standards for the field of cardiovascular imaging. The development of evidence, particularly effective risk stratification, guided growth, and set the standard for appropriate utilization practice which are in place in contemporary nuclear cardiology practice. Nuclear cardiology remains a leading imaging modality with millions of procedures performed each year in the US. Despite this history, recent downturns in utilization have been observed and for what once was a bounding success, nuclear cardiology now finds numerous hurdles that impinge on growth.

One challenge with nuclear cardiology has been the breadth of indications which historically have fueled imaging of the large population of patients with stable ischemic heart disease. The challenges facing nuclear cardiology in our current era have prompted researchers to focus on novel indications in varied fields including inflammatory and infective disorders. The greater the breadth of indications for any cardiovascular imaging modality, the more likely that utilization can be preserved

Reprint requests: Leslee J. Shaw, PhD, Emory university, Atlanta, USA; lshaw3@emory.edu

J Nucl Cardiol 2017;24:757.

1071-3581/\$34.00

Copyright (c) 2017 American Society of Nuclear Cardiology. to serve a more diverse patient population. In many ways, prior to these new applications for nuclear cardiology, the field has been stagnant with a failure to grow innovative options in cardiovascular imaging. The limited growth of cardiac PET is a great example of a modality which is poised to provide substantial benefit to nuclear cardiology but has unfulfilled expectations in terms of growth.

Innovation is at the heart of the development of diverse applications in nuclear cardiology, and the field must also focus on the coming together of high-quality imaging researchers. But, innovation is not just a new indication but is limitless in terms of advancing our field. Innovation can be at the local laboratory or at the national level. Where we are today should never constrain improvements in imaging practice and creating improvements to enhance patient care driven by nuclear cardiology. We, at the journal, would like to put forth a challenge to the nuclear cardiology community to embrace innovation and create an energy in our field that harkens to prior years when our field set standards for cardiovascular imaging.

If you have never set aside a day or an afternoon to contemplate innovating your practice, then you should! It is a daunting task and requires focus and patience. The field of nuclear cardiology needs to embrace innovation as the new standard for our imaging world. Where can we go from here is a statement that should be voiced daily? And, where we can go is not defined or limited but for our visions of innovation. If innovation is a key, then we need to start now and not be burdened by conventional practices but embrace discussions on improvement and futuristic topics. Investing our best thoughts in our field of nuclear cardiology, bringing the brightest leaders to develop newness, and creating an unimagined world for cardiovascular imaging is possible and will render our field not only relevant but at the forefront of novelty in imaging-guided care.

\section{Disclosure}

The authors have nothing to disclose. 